\title{
Plastic footwear for leprosy
}

\author{
N H ANTIA \\ The Foundation for Research in Community Health, 84-A, R.G. \\ Thadani Marg, Worli, Bombay 400 018, India
}

\section{Accepted for publication 25 August 1989}

\begin{abstract}
Summary The anaesthetic foot in leprosy poses the most major problem in the rehabilitation of its patients. Various attempts have been made to produce protective footwear such as the microcellular rubber-car-tyre sandals. Unfortunately these attempts have had little success on a large scale because of the inability to produce them in large numbers and the stigma attached to such unusual footwear. While such footwear may be superior to the 'tennis' shoe in protecting the foot from injury by the penetration of sharp objects, it fails to distribute the weight-bearing forces which is the major cause of plantar damage and ulceration in the anaesthetic foot. This can be achieved by providing rigidity to the sole, as demonstrated by the healing of ulcers in plaster of paris casts or the rigid wooden clog.

A new type of moulded plastic footwear has been evolved in conjunction with the plastic footwear industry which provides footwear that can be mass produced at a low price and which overcomes the stigma of leprosy. Controlled rigidity is provided by the incorporation of a spring steel shank between the sponge insole and the hard wearing plastic sole. Trials have demonstrated both the acceptability of the footwear and its protective effects as well as its hard wearing properties.
\end{abstract}

\section{Introduction}

Plantar ulceration poses the single greatest problem in the rehabilitation of leprosy patients. While a patient with facial or hand deformities can continue to work even in a limited manner, ulceration of the foot poses a far more difficult problem. Once damaged, the foot continues to ulcerate even with the trauma of limited locomotion. The unsightly swollen foot under smelly bandages not only carries a stigma and social repulsion, but also prevents the patient from reaching his work place and carrying out even relatively sedentary activities. Unlike facial and some hand deformities, till now surgery has had little to off er in the correction of plantar ulceration except sequestrectomy, debridgement and occasional skin graft or flap cover which also break down from subsequent trauma to the anaesthetic foot. 


\section{Materials and methods}

Care of anaesthetic feet and provision of footwear have been taught and extensively practised by patients, but with poor results outside the precincts of the leprosarium. The most effective means of healing plantar ulcers is not by antibiotics and dressing but bed rest, immobilization in a plaster of paris cast, or the use of a solid wooden clog. Unfortunately all of these have only proved to be temporary expedients. The car tyremicrocellular hand-sewn sandal is extensively employed within the leprosarium, and although an improvement on the ordinary 'chappal' or 'tennis' shoe, it still has several disadvantages. It cannot be produced in bulk and depends on the microcellular insole for softness, for providing protection from thorns and for providing a durable though flexible sole. As explained later, this non-rigid footwear does not provide the basic requirement for the distribution of pressures which is the chief cause of plantar damage. Another major handicap of this sandal is that it stands out from all normal footwear; being exclusively used by leprosy patients, it carries its own stigma which prevents most patients from wearing it in public outside leprosaria. The patient therefore prefers to wear the common tennis shoe to hide the deformity and to avoid being conspicuous as a sufferer of leprosy.

The normal foot, even in barefoot walking, is protected from injury by sensation provided to the sole by the median and lateral plantar branches of the posterior tibial nerve. This protection is not only against sharp objects, like thorns and nails, as is commonly believed, but more important, pain prevents the build-up of excessive pressures generated under bony pressure points in the foot which is the major cause of damage in feet that do not have normal protective sensation. Ernest Price as early as 1959, in a WHO workshop on Deformities in Leprosy held at Karigiri, demonstrated this by charting the frequency of the sites of plantar ulceration in the leprous foot; this followed a regular pattern along the weight-bearing areas of the foot, the most common site being under the ball of the great toe where pressure reaches its height during the take-off thrust when walking and running. With his customary simplicity and elegance he also demonstrated the difference in pressures in the normal foot as compared to that in the feet of leprosy patients at various stages of sensory loss or damage using an inked paper for footprint impressions. This has been published in detail in his Chapter on 'Care of the Feet' in the second edition of Leprosy in Theory and Practise. ${ }^{1}$ In this he also describes the preulcerative stage where 'the degree of anaesthesia may be minimal'; this is caused by aseptic traumatic fat necrosis due to unconscious build-up of excessive pressure; this ultimately results in scarring of the deeper tissues, which in turn makes the area susceptible to even lesser trauma, and leads ultimately to frank ulceration and infection which may eventually result in osteomyelitis and necrosis of the underlying bone.

Another factor which further predisposes the anaesthetic foot to ulceration is the intrinsic muscle paralysis associated with nervedamage. This results in the claw deformity of the toes and shift of the protective sesamoid bones from under the head of the first metatarsal. ${ }^{2}$ Drop foot also results in maldistribution of pressures to the anterior part of the foot.

The reason that plantar ulcers heal with rest and elevation in a plaster of paris cast or the wearing of wooden clogs demonstrates the importance of distribution of pressures on a larger surface of the foot, preventing the building of excessive pressures under bony points in a supple foot which does not have normal protective sensation. While the plaster 
cast and rigid rocker may be ideal for the distribution of pressures, they are impractical for everyday use because of their total rigidity which makes walking difficult and because of their unacceptable appearance. It is therefore important to devise a method which permits controlled rigidity with a certain degree of rocker action as well as some degree of flexibility.

An ideal footwear should have the following requirements:

(a) Above all it should protect the feet from the major known causes of ulceration, i.e. by distribution of pressure and preventing injury by sharp objects.

Besides this, it should also have the following qualities:

(b) It should be aesthetically acceptable and not carry the stigma of leprosy.

(c) It should be mass produced at reasonable cost and be readily bought off the shelf in various sizes like any other footwear; preferably it, or a similar model, should be used by the general population.

(d) Can be modified in its external shape and colour at intervals to prevent the development of stigma.

(e) Should be hard wearing and usable on different terrain.

(f) Easy to repair, preferably by the patient himself/herself.

(g) Quick wearing components should be replaceable and purchased readily.

(h) Should be water resistant and impervious to secretions from ulcers.

(i) Easy to clean.

(j) Should be comfortable to wear.

Bombay is one of the largest centres for the production of plastic footwear, and the author decided to explore the possibility of utilizing the locally available expertise and facilities to devise a suitable footwear for leprosy patients which would incorporate some or all of the abovementioned criteria. Through a prolonged process of trial and error and utilizing the contacts with various sections of this industry, the following footwear has been evolved which meets many if not all of the requirements.

\section{Description of footwear}

This shoe consists of three components (Figures 1, 2(a), (b) and 3).

1 Single unit extruded hard wearing plastic sole together with the uppers. This extruded shoe has the shape of some of the normal sandals sold in the market but has the following modifications.

(a) Thicker sole for longer wearing.

(b) Permits concealing of moderate deformities of the foot and toes.

(c) Has enough ventilation to prevent maceration of the skin.

(d) A heel counter to prevent lateral mobility.

(e) Increased depth to accommodate an $8 \mathrm{~mm}$ thick sponge insole.

(f) A depression to accommodate the steel shank.

2 Steel shank. A spring steel shank $240 \times 40 \mathrm{~mm}$ and $1 \mathrm{~mm}$ thick with a curve commensurate with the curve of the sole is fitted in the depression on the superior surface of the plastic sole underneath the sponge insole. After a process of trial and error the 

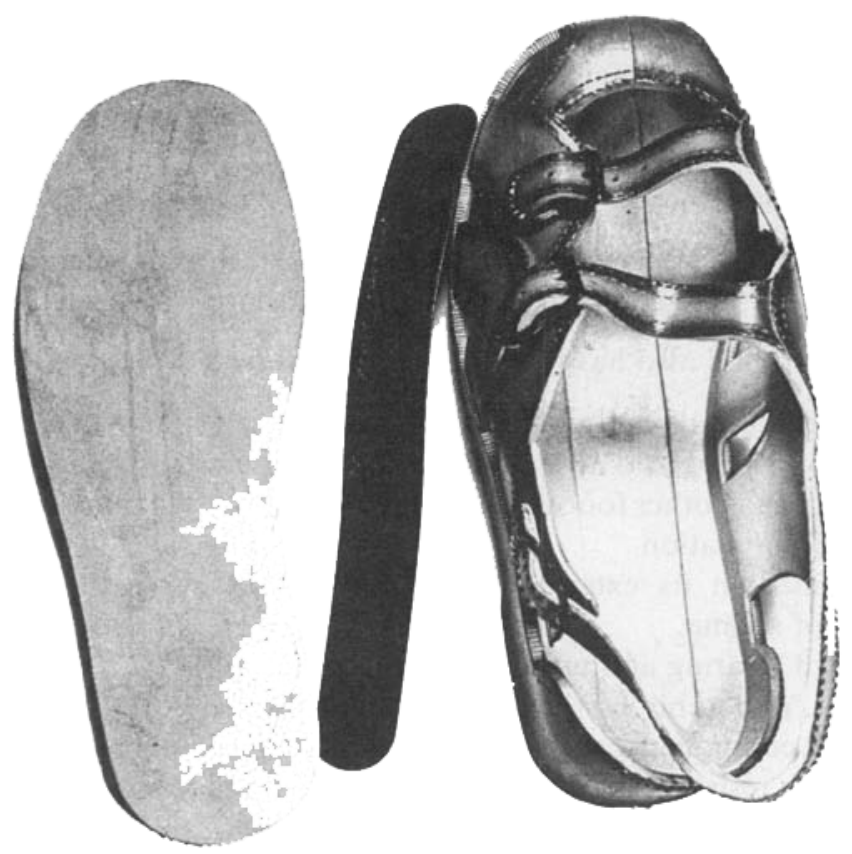

Figure 1. Components of footwear. From left to right: EVA insole, steel shank and view of the footwears plastic uppers showing the depression for the steel shank on the superior surface of the plastic sole.

present shank has been found to provide adequate rigidity to the sole for distribution of pressures and yet permits a degree of flexibility for comfortable locomotion. It is virtually indestructible and also prevents injury by thorns and nails.

3 Closed cell 18 shore plastic insole. This $8 \mathrm{~mm}$ thick EVA insole available in the market is impervious to water and secretions, provides adequate cushioning and is more durable than softer sponges used in normal footwear or the traditional microcellular sponge. The semirigid shank further distributes pressures on this insole and reduces its wear under pressure points. The insole is the least durable part of the shoe and can be purchased as an accessory which can be slipped in and, if necessary, glued into the shoe by the patient himself.

The report of the earlier model of the shoe tested at the Kondhwa Leprosy Hospital (to be published shortly) revealed acceptability to both male and female patients but had to be modified to provide a heel counter and thicker and better designed straps to prevent their breakage. The thicker straps avoiding sharp angles in the later models have not only overcome this flaw but can be readily repaired by the patient himself or the street cobbler by simple heat-jointing with a heated metal strip. The six month trial of the shoe at Kondhwa demonstrated not only the protection of healed ulcers but also the healing of moderate ulcers when the shoes were worn without any dressing.

This long lasting shoe at the modest price of US $\$ 10$ per pair meets most of the requirements for leprosy footwear and we hope will prove to be a major advance in the rehabilitation of leprosy patients. We feel it could be worn with advantage by all leprosy patients, for every leprosy patient has the risk of potential damage due to nerve 


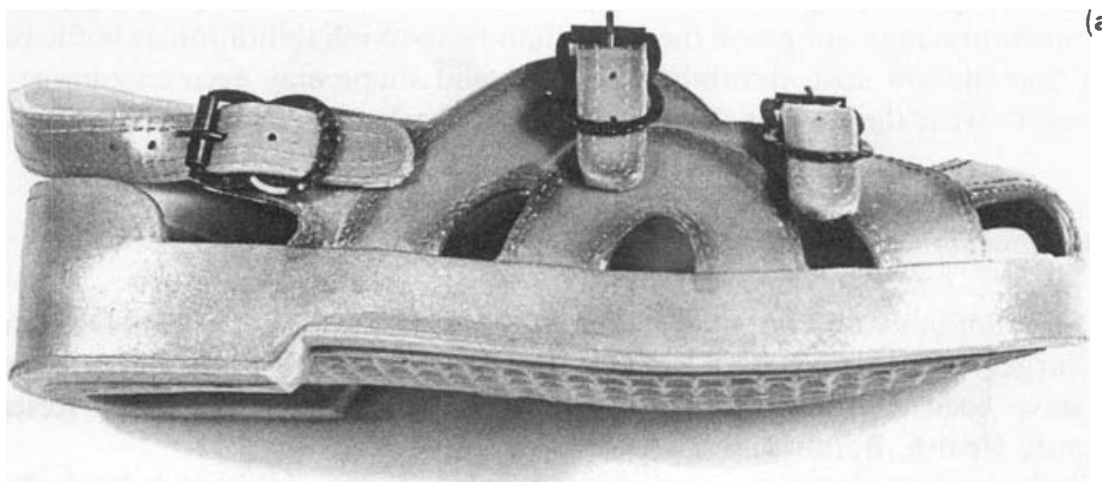

(a)

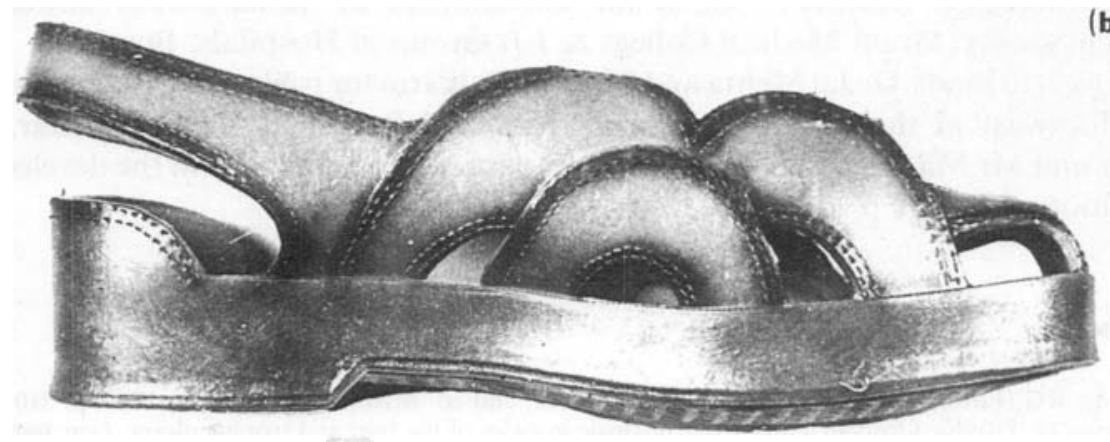

(b)

Figure 2(a) and (b). Lateral views of the footwear showing the deformity-concealing yet ventilating pattern of the uppers.

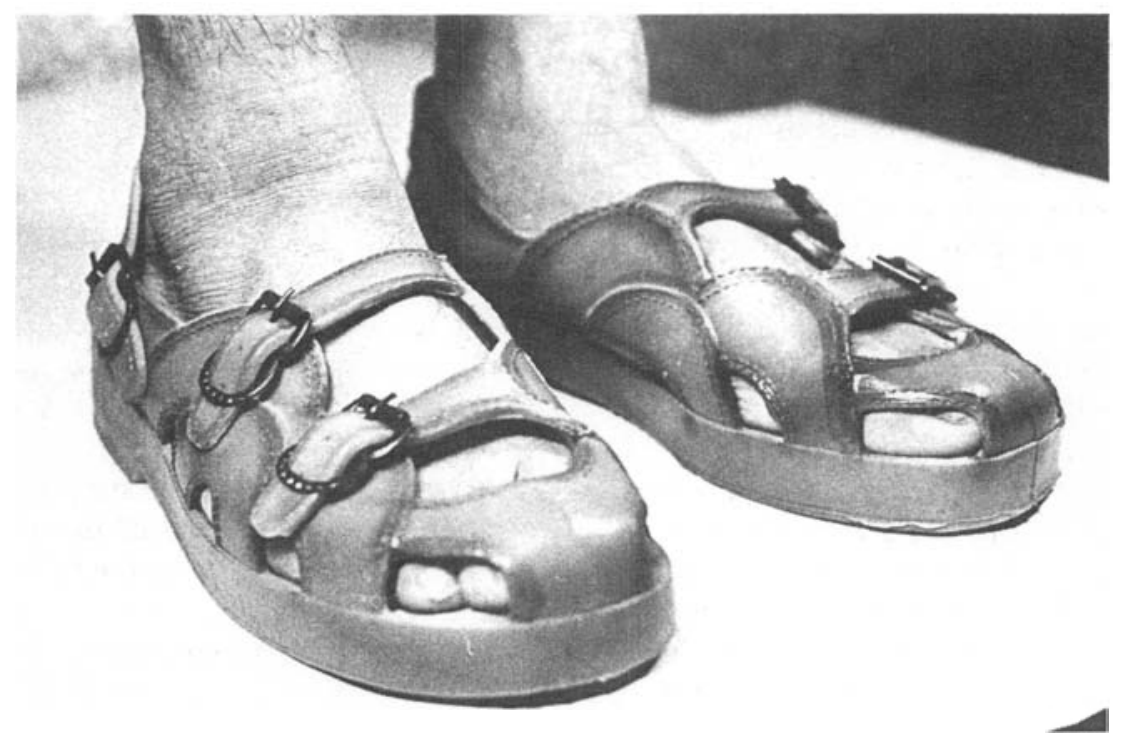

Figure 3. View of footwear when worn. 
involvement. It is also hoped that in association with the newer forms of nerve release, plantar ulceration may not prove the major handicap to rehabilitation as in the past. It is also felt that the low cost, durability, comfort and shape may even encourage normal individuals to wear these shoes if sold in the open market, thus improving acceptability.

\section{Acknowledgments}

The early part of this study was undertaken by the author while at the Tata Department of Plastic Surgery, J.J. Group of Hospitals, Bombay. After the author's retirement, the studies have been conducted in co-operation with the Foundation For Research in Community Health, Bombay.

We wish to acknowledge the support provided for a part of this study by the Scientific and Technological Research Cell of the Government of Maharashtra, through the Research Society, Grant Medical College \& J.J. Group of Hospitals, Bombay.

We wish to thank Dr Jal Mehta and Mr V N Kulkarni for trials of the different models of the footwear at the Kondhwa Leprosy Hospital, Pune and Dr M Uplekar, Dr S Arolkar and Mr Madhusudan Rokle for their participation and help in the development of this footwear.

\section{References}

1 Cochrane RG (Editor). Leprosy in Theory and Practice, 2nd ed. Bristol: John Wright, 1964; p. 510.

${ }^{2}$ Srinivasan H. Trophic ulcers in leprosy II-intrinsic muscles of the foot and trophic ulcers. Lepr India, 1964; 36: $110-18$. 